\title{
Identification and geographical distribution of pyrethroid resistance mutations in the poultry red mite Dermanyssus gallinae
} Evangelia Katsavou ${ }^{1}$, Spyros Vlogiannitis $^{1}$, Eleanor Karp-Tatham ${ }^{2}$, Damer P. Blake ${ }^{2}$, Aris llias ${ }^{3}$,
Christina Strube ${ }^{4}$, Ilias Kioulos ${ }^{1}$, Wannes Dermauw ${ }^{5}$, Van Leeuwen Thomas ${ }^{5 *}$, John Vontas ${ }^{1,3^{*}}$

\footnotetext{
${ }^{1}$ Laboratory of Pesticide Science, Department of Crop Science, Agricultural University of Athens, 75 lera Odos Street, GR-11855 Athens, Greece

2 Pathobiology and Population Sciences, The Royal Veterinary College, Hawkshead Lane, Hatfield, Hertfordshire, AL9 7TA, United Kingdom

${ }^{3}$ Institute of Molecular Biology \& Biotechnology, Foundation for Research \& Technology Hellas, 100 N. Plastira Street, GR-700 13 Heraklion, Crete, Greece

${ }^{4}$ Institute for Parasitology, Centre for Infection Medicine, University of Veterinary Medicine Hannover, Buenteweg 17, 30559 Hanover, Germany

${ }^{5}$ Laboratory of Agrozoology, Department of Plants and Crops, Faculty of Bioscience Engineering, Coupure Links 653, Ghent University, B-9000 Ghent, Belgium
}

Keywords: poultry red mite; Dermanyssus; pyrethroid resistance; diagnostics; resistance origin and spread

*corresponding authors: Thomas.VanLeeuwen@ugent.be or vontas@imbb.forth.gr 


\begin{abstract}
BACKGROUND: The poultry red mite (PRM) Dermanyssus gallinae is the most common ectoparasite on poultry and causes high economic losses in poultry farming worldwide. Pyrethroid acaricides have been widely used for its control and, consequently, pyrethroid resistance has arisen. In this study we aim to investigate the occurrence of resistance and study the geographical distribution of pyrethroid resistance mutations across PRM populations in Europe.

RESULTS: Full dose-response contact bioassays revealed very high levels of resistance against several pyrethroids ( $\alpha$-cypermethrin, fluvalinate and cyfluthrin) in two PRM populations from Greece, compared to a susceptible reference strain. Resistance was associated with mutations in the gene encoding the target-site of pyrethroids, the voltagegated sodium channel (VGSC). Mutations, M918L and L925V in domain IIS4-S5 and F1534L in domain IIIS6, were found at positions known to play a role in pyrethroid resistance in other arthropod species. Subsequent screening by sequencing VGSC gene fragments IIS4-S5 and IIIS6 revealed the presence and distribution of these mutations in many European populations. In some populations, we identified additional or different mutations including M918V/T, L925M, T929I/F, 1936F and F1538L. The latter mutation is a possible alternative for F1538I that has been previously associated with pyrethroid resistance in other mites and ticks.
\end{abstract}

CONCLUSION: We report very high levels of pyrethroid resistance in PRM populations from Greece, as well as the identification and geographical distribution of ten pyrethroid resistance mutations in PRM populations across Europe. Our results draw attention to the need for an evidence-based implementation of PRM control, taking acaricide resistance management into consideration. 


\section{INTRODUCTION}

The poultry red mite (PRM), Dermanyssus gallinae (De Geer, 1778), is recognized as the most important blood sucking ectoparasite of laying hens and broiler birds worldwide. It poses an important threat to animal welfare but, to a lesser extent, also to public health ${ }^{1}$. A PRM infestation affects birds by causing feather pecking, anaemia, restlessness, aggression, reduced egg production (increased fragility of the egg shell), blood staining of the eggs or transmission of pathogenic agents. ${ }^{1,2}$ The economic impact of PRM was approximately 130 million euros in 2004 and increased to an estimation of 231 million euros in $2017 .{ }^{3}$ Over $80 \%$ of European layer farms are infested with PRM. More specifically, more than $90 \%$ of Dutch, German and Belgian layer farms and most Northern Greek farms are infested with PRM. 4,5 PRM infestations have also been reported in China, Japan, Australia and Brazil. ${ }^{6}$ The high PRM infestation rates in Europe may be associated with recent legislation for the transformation of housing systems in laying hen husbandry in EU member countries, as well as the withdrawal of many acaricide products from the market, due to strict legislation and safety concerns. ${ }^{3}$

The control of PRM populations is primarily based on acaricide applications (sprays, mists and dusts) and a small number of alternative options, including biological control (e.g. acaripathogenic fungi, predatory mites) and biopesticides (e.g. plant extracts). ${ }^{7}$ Few acaricide products are however registered for the control of PRM in Europe, depending on the local market and legislation, including organophosphates (phoxim), pyrethroids (cypermethrin, permethrin, deltamethrin), carbamates (bendiocarb), amidines, isoxazolines (fluralaner), abamectin and spinosad..$^{3,8,9}$

The extensive use of these acaricides and the life history traits of PRMs (short life cycle and high fecundity) have resulted in the development of acaricide resistance. There have been many reports of PRM resistance against acaricides, mainly for pyrethroids: Italy in 1984 and 2009 (permethrin) $^{10,11}$; former Czechoslovakia in 1985 (a-cypermethrin) ${ }^{12}$; France in 1996 (permethrin) ${ }^{13}$; Sweden in 2000 (permethrin) ${ }^{14}$ and Poland in 2010 ( $\alpha$-cypermethrin) $)^{15}$. Other unsuccessful attempts of PRM control using acaricides were reported in Germany, UK and Hungary. ${ }^{16}$ 
Pyrethroid resistance has been investigated at the molecular level in Acari and can be metabolism-based, i.e. faster or more efficient detoxification of the active ingredient that reduces target exposure. This has been inferred indirectly as shown by synergism experiments, but also metabolism assays. ${ }^{17,18}$ On the other hand, resistance has also been linked to alterations in the target site of pyrethroids, the voltage-gated sodium channel (VGSC). ${ }^{19,20}$ A number of VGSC mutations have been characterised to-date in several Acari and insect species, including those located at transmembrane segments 4 to 5 of the domain II (IIS4-IIS5) region of the VGSC gene at amino acid positions M918, L925, T929 and 1936, as well as mutations located in IIIS6, at amino acid positions F1534 and F1538 (Figure 1) (Musca domestica numbering). ${ }^{19}$ In the cockroach, Blatella germanica, F1538I has been associated with a very strong pyrethroid insensitivity phenotype ${ }^{21}$ and this mutation has been found to be common in mites and ticks, such as Tetranychus urticae and Rhipicephalus microplus. ${ }^{22-24}$ On the other hand, $\mathrm{M918T}$, also known as the super-kdr mutation and known to confer strong resistance against pyrethroids ${ }^{19}$, has been identified in the VGSC of pyrethroid resistant spider mite species. ${ }^{25,26}$ Finally, certain mutations appear to enhance the pyrethroid resistance phenotype conferred by others, rather than being directly causal, while it is also possible that they play a role in improving the fitness of the mutated allele. ${ }^{19,27}$

Molecular mechanisms of pyrethroid resistance have not yet been investigated in PRM but the recently released PRM whole genome sequence ${ }^{28}$ now provides a unique opportunity to investigate acaricide resistance mechanisms in this mite species. In this study, we associated pyrethroid resistance phenotypes with mutations in the VGSC genotypes and surveyed these mutations in European PRM populations.

\section{MATERIALS AND METHODS}

\subsection{PRM populations}

The susceptible PRM strain (SUSC) is a laboratory strain, that has been maintained at the Institute for Parasitology of the University of Veterinary Medicine Hannover (Germany) and has not been exposed to acaricides for several decades. ${ }^{29}$ The GRC and BEL samples of PRM were collected in 2018 from 6 locations around Athens, Greece and 2 locations in Belgium respectively (Table 1). The GRC1, GRC2, GRC5 strains were collected from heavily sprayed 
poultry farms near Athens (Megara and Ilion), while the GRC6 and the BEL1, BEL2 strains were sampled from private housing coops in Greece and Belgium, respectively. Additional samples were collected from different European regions, primarily from large poultry farms, with acaricide application history. The mites were collected either alive in plastic boxes containing mites of all developmental stages (samples from Greece, Belgium and Germany) or in ethanol (the remaining European populations).

\subsection{Bioassays}

Laboratory tests to assess pyrethroid susceptibility were conducted by exposing mites in treated glass vials. Technical grade pesticide was dissolved in acetone, diluted and $0.5 \mathrm{~mL}$ of solution was transferred into individual $20 \mathrm{~mL}$ glass vials. Vials were rolled on their sides until the acetone evaporated, leaving a uniform film of acaricide on the inner surface. Technical grade $\alpha$-cypermethrin, cyfluthrin and fluvalinate were obtained from Sigma Aldrich. Approximately ten mites (3 day-starved deutonymphs) were placed in each vial, after which it was kept in an incubator at $25{ }^{\circ} \mathrm{C}$ for $20 \mathrm{~h}$. After the treatment, mites were evaluated individually and considered dead in absence of leg movement when prodded. Toxicity data were analyzed using PoloPC (LeoRA, Software, Berkeley, CA).

\subsection{DNA \& RNA extraction, amplification and sequencing}

Genomic DNA was extracted from pools of approximately 50 mites per sample, using DNAzol reagent (Molecular Research Center, Inc.) according to the manufacturer's instructions. The mites were collected alive (GRC, GER and BEL samples) or washed from ethanol with distilled water (remaining EU samples) before further treatment. Pooled mites were grinded with pestles in $500 \mathrm{ul}$ DNAzol in $1.5 \mathrm{ml}$ Eppendorf tubes. The samples were centrifuged at approximately $10,000 \mathrm{~g}$ for $10 \mathrm{~min}$ at room temperature. The supernatant was transferred in a new tube and mixed with 250 ul of $100 \%$ ethanol. After 20 min centrifugation at approximately $13,000 \mathrm{~g}$ at room temperature, the supernatant was discarded, and the pellet washed with $1 \mathrm{ml}$ of $75 \%$ ethanol. Then the samples were centrifuged for $5 \mathrm{~min}$ at approximately $13,000 \mathrm{~g}$ at room temperature, the supernatant was discarded, and the pellet was air dried and finally dissolved in 20 ul sterile water.

Two fragments of the VGSC were amplified: domain IIS4-S5 (145 bp) and domain IIIS6 (237 bp). PCR reactions were initiated with approximately $200 \mathrm{ng}$ of gDNA as template, in $1 \times$ Taq 
reaction buffer (Promega, SB Biotechnology Suppliers S.A. Athens, Greece), using 2 pairs of primers (KF1 5'-CAAGTCATGGCCGACGTTGA-3', KR1 5'-GTCGGTGTAGTTCTTGCCGAA-3' for the amplification of IIS4-IIS5 and KF3 5'-AAAAGACGACCAGCCCGACT-3', KR3 5'GGTATGGCTTTGGCGGGTTT-3'for the amplification of IIIS6) with a final concentration of 2 $\mathrm{mM} \mathrm{MgCl} 2$ and $0.5 \mathrm{mM}$ of each primer. The $\mathrm{PCR}$ reactions were performed under the following conditions for both primer pairs: $94^{\circ} \mathrm{C}$ for $3 \mathrm{~min}$, then 35 cycles of $95^{\circ} \mathrm{C}$ for $30 \mathrm{sec}$, $56{ }^{\circ} \mathrm{C}$ for $30 \mathrm{~s}, 72{ }^{\circ} \mathrm{C}$ for $30 \mathrm{sec}$, followed by a final cycle of $72{ }^{\circ} \mathrm{C}$ for $2 \mathrm{~min}$. Primers were designed based on the VGSC gene sequence from the recently published PRM genome ${ }^{28}$ and used to PCR amplify partial sequences of the PRM VGSC gene. PCR products were purified using NucleoSpin Extract II (Macherey-Nagel) and directly sequenced from both sides with the original PCR primers. Sequencing reactions were performed at CeMIA SA (University of Thessaly, Greece). Sequence data were analysed using Bioedit 7.0.1 ${ }^{30}$ and compared to the sequence of the susceptible strain (SUSC). For the SUSC, GR1, GR2 populations, the IIS6 domain was amplified additionally to the IIS4-IIS5 and IIIS6. Total RNA was extracted from mass homogenates of PRM using TRI reagent (Sigma-Aldrich, Bornem, Belgium) according to the manufacturer's instructions. The RNA was treated with DNase (Turbo DNase, Ambion) and $2 \mu \mathrm{g}$ RNA for each sample was reverse transcribed into CDNA using SuperScript ${ }^{\mathrm{TM}}$ III Reverse Transcriptase (Invitrogen) and oligo(dT) ${ }_{20}$. One pair of primers was used for the amplification of the VGSC domains IIS4-IIS6 (430 bp) (KF1 5'-CAAGTCATGGCCGACGTTGA-3' and KR4 5' CTCCTGCAGCTTCTTCGTGT 3'). The PCR reaction was performed with the same reagent concentrations as above mentioned, and under the following conditions: $94{ }^{\circ} \mathrm{C}$ for 3 min, then 35 cycles of $95{ }^{\circ} \mathrm{C}$ for $30 \mathrm{sec}, 58{ }^{\circ} \mathrm{C}$ for $30 \mathrm{~s}, 72{ }^{\circ} \mathrm{C}$ for $30 \mathrm{sec}$, followed by a final cycle of $72{ }^{\circ} \mathrm{C}$ for $2 \mathrm{~min}$. Sequencing reactions and analysis were performed as described previously.

\section{RESULTS}

\subsection{Characterization of pyrethroid resistance in GRC1, GRC2 and the susceptible strain SUSC}

We have first investigated pyrethroid resistance in two PRM populations, GRC1 and GRC2, collected in 2018 from large poultry houses near Athens (Megara and Ilion, Greece, respectively). The GRC1 population exhibited high levels of resistance to $\alpha$-cypermethrin, cyfluthrin and fluvalinate (resistance ratio $>1,000,000$-fold) while the GRC2 population 
exhibited high levels of resistance to $\alpha$-cypermethrin, compared to the susceptible strain SUSC (Table 1). The $\mathrm{LC}_{50}$ of $\alpha$-cypermethrin, cyfluthrin and fluvalinate in the GRC1 population exceeded $1000 \mathrm{mg}$ a.i./L, and the $\mathrm{LC}_{50}$ of $\alpha$-cypermethrin in the GRC2 population was higher than $2000 \mathrm{mg}$ a.i./L. In contrast, the $\mathrm{LC}_{50}$ of the susceptible laboratory strain was determined at $5.84 * 10^{-4} \mathrm{mg}$ a.i./L for $\alpha$-cypermethrin, $8.76 * 10^{-5} \mathrm{mg}$ a.i./L for cyfluthrin and $1.76^{*} 10^{-4} \mathrm{mg}$ a.i./L for fluvalinate (Table 1$)$.

\subsection{Identification of target site mutations associated with pyrethroid resistance}

The sequences of VGSC domain II (S4-S5-S6) and III (S6) were obtained from the recently available PRM genome sequence ${ }^{28}$. None of the previously characterised pyrethroid resistant mutations (Figure 1A) were present in domain II S4-S5 and III S6 of the VGSC from the susceptible strain SUSC. Subsequently, the respective sequences from the GRC1 and GRC2 populations that exhibited high resistance against pyrethroids were obtained and compared with the SUSC strain. Non-synonymous mutations were detected at codons 918, 925 (domain II) and 1534 (domain III) in both GRC1 and GRC2 (M. domestica numbering, underlined triplets and deduced amino acids in Figure $1 \mathrm{~A}$ ), at positions which have been associated with pyrethroid resistance in other species. In the sample of pooled mites, both M918L and F1534L appeared fixed in the GRC1 population, while M918L and L925V appeared fixed in the GRC2 population, which showed the strongest resistance phenotype. We did not observed any mutations in domain IIS6 in both GRC1 and GRC2, nor the SUSC strain. We use the term 'fixed' when sequencing chromatographs do not show any evidence of an alternative nucleotide at the position of interest, based on sequencing a PCR fragment amplified on pooled material. This does not necessarily mean complete fixation at the population level, as a large number of single mites needs to be assayed to support such claims. This limitation in assessing frequency estimates also implies for pooled samples in 3.3.

\subsection{Monitoring the presence and frequency of pyrethroid resistance mutations in PRM populations across Europe}

We subsequently investigated the presence of M918L, F1534L and L925V, as well as possible additional pyrethroid resistance mutations, across PRM populations from Europe. Fiftythree samples from 15 European countries were examined in total: Greece, Italy, France, Spain, Portugal, UK, Ireland, Czech Republic, Belgium, Netherlands, Denmark, Romania, 
Albania, Croatia, Germany and Turkey (Figure 2). More specifically, we analysed by direct sequencing of PCR products two fragments of the VGSC gene, parts of the domains II (IIS4IIS5) and IIIS6. Resistant mutations were detected as double peaks (not fixed) or single peaks (fixed), indicating the presence of a mixture of resistant and susceptible alleles or fixed resistant/susceptible alleles only in each sample, respectively. But see the limitation in terminology outlined above.

Except for the SUSC strain and the samples GER1 (Hannover, Germany) and GBR10 (West Sussex, UK) that bear no mutations in the examined domains, 50 out of 53 samples analysed had at least one mutation at amino acid positions previously associated with pyrethroid resistance (Table 2). Overall, eight putative pyrethroid resistance mutations were identified across all PRM populations at positions M918 (918L, 918V, 918T), L925 (925M, 925V), L929 (925V and 925M), I936 (936F) in domain II and two additional ones, F1534L and F1538L in domain III (Figure 1B). The M918L substitution was the most frequently found, as it was identified in 37 out of 53 samples (69.8\%) and the amino acid leucine was found to be the predominant allele in our samples at the 918 position. In contrast, the amino acid valine was identified at codon 918 (M918V) in only one out of 53 samples (NLD2, Netherlands) and amino acid threonine (M918T) was also rare (4 out 53 samples). L925V was also frequently detected in 19 out of 53 samples (35.8\%), in contrast to methionine at the same position (3 out of 53 samples). In general, the rarest substitutions were T929I and I936F in 4 samples each (7.5\%) of the total. Two additional mutations were found in domain III, F1534L and F1538L (Table 2 and Figure 1A). F1534L was the most frequent mutation, as it was present in 31 out of 53 samples (58.5\%). F1538L was found only in 3 samples (5.7\%): two derived from the UK (GBR3, GBR9) and one from France (FRA).

Focusing on the country level, PRM populations from three countries - Greece, Croatia and Albania - appear to have the same VGSC gene mutation profile, as in all of these populations M918L, L925V and F1534L were present (Figure 3). Samples originating from Portugal (PRT1, PRT2, PRT3, PRT4) and Spain (ESP1, ESP2, ESP3) also had a similar profile, as they all showed a combination of M918L with F1534L. The population collected from France (FRA) had the M918T and F1538L substitution. Additionally, in populations from Italy (ITA1, ITA2) we identified two mutations, M918L and L925V. The sample from Turkey (TUR) was the only population bearing the T929I mutation alone. T929I was also present in Romania (ROU1, 
ROU3, ROU4, ROU5, ROU6), along with M918L and F1534L. In samples from Czech Republic (CZE1, CZE2) M918L, L925M/V and 1936F were present. In the Netherlands, all three most commonly found mutations were identified - M918L, L925V and F1534L - while M918V was only found in only one sample. Belgian samples appeared to have M918L, L925M, 1936F and F1534L. The samples obtained from Denmark (DNK1, DNK2) harboured both M918L and L925V. Last, the highest diversity of mutations was found in UK samples: M918L/T, L925V/M, 1936F, F1534L and F1538L. In addition, in two UK samples (GBR7, GBR8) we identified a point mutation (TTT to CTT, not fixed) at the 1537 position (phenylalanine to leucine, both hydrophobic amino acids), previously mentioned as a 'pyrethroid sensing residue ${ }^{31}$, suggesting it might require further investigation, as it was combined with already known resistance mutations.

\section{DISCUSSION}

High levels of pyrethroid resistance were detected in field populations (GRC1, GRC2) from Megara and Ilion respectively (Greece), where almost $40 \%$ of the country's hen rearing is hosted. Resistance against $\alpha$-cypermethrin, cyfluthrin and fluvalinate pyrethroids, acaricides which have been used extensively in Greece for several years, scaled over 10,000-fold, compared to a susceptible laboratory strain SUSC (Table1).

A M918L mutation in domain IIS4-S5 of the VGSC gene was at very high frequency, looking fixed in the pooled sample of the GRC1 strain. M918L is a variant of the well-known super$k d r$ mutation (M918T) ${ }^{19,32}$ and has also been found in the spider mite $T$. urticae ${ }^{26}$, while a M918T mutation has been associated with pyrethroid resistance in T. evansi. ${ }^{25}$ The M918L mutation alone confers moderate levels of resistance ${ }^{33}$, but when combined with other mutations, particularly at position L925 $^{34-37}$ provides higher resistance levels. A F1534L mutation was also present in GRC1. An alternative mutation at the same position, F1534S, has been identified in multi-resistant $T$. urticae population on different crops. ${ }^{26}$ On the other hand, a F1534C mutation has been identified in pyrethroid resistant mosquitoes ${ }^{38,39}$ and upon functional characterization in Xenopus oocytes was shown to confer increased levels of pyrethroid insensitivity. ${ }^{40}$

Together with M918L, an L925V mutation appeared was fixed in the GRC2 population, which probably showed even higher levels of pyrethroid resistance $\left(L_{50}>2000 \mathrm{mg}\right.$ a.i./L). 
The L925V mutation, and a variant thereof (L925I) has been associated with resistance in several European populations of Varroa destructor, a mite belonging to the same superfamily (Mesostigmata: Dermanyssoidea) as $\mathrm{PRM}^{41-43}$, but also in others arthropod species, including ticks and bedbugs. ${ }^{19,44,45}$

We subsequently assessed the presence and frequency of pyrethroid resistance mutations in a large number of PRM populations across Europe (53 samples in total from 15 European countries), aiming to investigate the presence and geographical distribution of M918L, F1534L and L925V, as well as possibly additional mutations, in several countries where pyrethroid resistance cases had already been reported. ${ }^{1,11,13,16}$ Pyrethroid resistance mutations were widely spread across Europe, as only 3 out of 53 examined samples did not have any mutation in the VGSC domains (IIS4-S5 and IIIS6) that were investigated in this study. M918L and F1534L, mutations that were associated with the striking pyrethroid resistance phenotype in the GRC1 and GRC2 populations from Greece, were the most common mutations across Europe (37 out of 53 and 31 out of 53 samples, respectively). In some cases, the M918L and F1534L mutations were, like in the GRC1 population, detected in combination and both fixed in field populations from Portugal (PRT1, PRT2), UK (GBR2) and Romania (ROU21). In other cases, the M918L and the L925V mutations were, like in the GRC2 population, both present and fixed in populations from Greece (GRC3, GRC5, GRC6), UK (GBR1) and Italy (ITA2).

A F1538L mutation in domain III, which has been associated with pyrethroid resistance in the pollen beetle Meligethes aeneus ${ }^{46}$, was detected in two samples originating from the UK and one from France. The role of a similar mutation, F1538I, in pyrethroid resistance has been confirmed by electrophysiological studies ${ }^{21}$ and was shown to confer very high levels of resistance to a number of pyrethroids in mites and ticks. ${ }^{24,47,48}$ In T. urticae, for example, F1538I was introgressed into a susceptible population and conveyed a strong pyrethroid resistance phenotype. ${ }^{23}$ Since both isoleucine and leucine amino acids are branched-chain amino acids and have a similar structure and physicochemical properties, the effect of the alternative mutation F1538L identified in PRM is likely to be equivalent to the effect of F1538I. However, the role of this mutation has to be functionally validated, alone or in combination with the other mutations identified in PRM. 
Interestingly, we also identified a F1537L mutation in two PRM samples from the UK (GBR7 and GBR8). Although position F1537 has been characterized as a pyrethroid sensing residue (also known as residue $\mathrm{F}_{31} 16^{31}$, the $\mathrm{F} 1537 \mathrm{~L}$ has not yet been validated as a resistance mutations in any species so far. However, its position right next to the major pyrethroid resistance mutation $\mathrm{F} 1538 \mathrm{I}$ in mite and tick VGSC genes may indicate its possible role in pyrethroid resistance and might warrant further investigation.

The M918T mutation, the well-known super-kdr mutation that has been reported in $T$. evansi and numerous other pyrethroid resistant arthropod species ${ }^{19,25}$, was also found in GBR9 from the UK and together with F1538L was fixed in the population. This finding might indicate the presence of even more intense and broad pyrethroid resistance phenotypes in the UK.

Another mutation, T929I, was detected and fixed in only one sample (TUR), in Turkey. T929I has been found in other species ${ }^{49}$. It has been functionally characterized in Xenopus and was shown to confer a drastic reduction of deltamethrin sensitivity 47,48 .

Although the sequencing/genotyping approach chosen in this study does not allow the identification of specific alleles, the co-occurrence of some mutations in the same allele was indirectly confirmed, as these mutations were found to be fixed in some populations. More specifically, the mutations M918L and F1534L were fixed in samples from Belgium, Greece, Portugal, Romania and UK, and thus presumably present on the same allele; the mutations M918L and L925V were fixed in a few samples originating from Greece, Italy and UK; the mutations M918T and F1538L were fixed in only one sample from the UK. As PRM is transmitted by dispersion between farms (on crates, egg trays or even humans) or, to a lesser extent, possibly by direct contact between birds $s^{50}$, it is likely that combinations of pyrethroid resistance mutations on certain alleles have been selected in one place as a single event, but subsequently spread across countries. This highlights the importance of passive transportation of PRM carrying resistance mechanisms, facilitated by human activities, which unfortunately promotes resistance spread at the world scale, as has been described in other species. ${ }^{51}$ This is also supported by similar mutation combinations found in neighbouring countries, as described above. Nevertheless, the precise worldwide haplotypic diversity, and thus the possible origin and expansion of resistant alleles, needs to be further investigated. 
Further studies are needed to better clarify the exact role of the reported mutations in resistance and the precise risk each allele possesses, as it is known from other species that the effect of certain mutations on the pyrethroid resistance phenotype, alone or in combination, largely varies in specificity, intensity and fitness. ${ }^{19,27}$

In addition, the possible contribution of additional non-target site resistance mechanisms in the striking resistant phenotypes observed in PRM cannot be excluded. Indeed, the combination of cytochrome P450 monooxygenase mediated metabolic resistance and target site resistance against pyrethroids has been found to be the most operationally significant in many species. ${ }^{27}$ The complexity of metabolic resistance may not facilitate the full understanding of detoxification pathways in the near future but at least some key candidate genes could now be identified, based on the recently acquired PRM genome. ${ }^{28}$

Overall, the results obtained on the pyrethroid resistance status of European PRM populations and the wide distribution of resistant mutations and genotypes across several countries, as well as the apparent ease of PRM transmission between farms ${ }^{50}$, raise an animal welfare alarm in Europe. These results draw attention to regulate the use of pyrethroids, as well as additional acaricides for the reduction of PRM, taking acaricide resistance management into consideration. The development of DNA markers and the use of diagnostic tools will substantially facilitate screening and continuous monitoring of pyrethroid resistance alleles in poultry farms, providing critical evidence for resistance management, in order to limit further spreading of resistance and maintain the effectiveness and sustainability of current control tools.

\section{AKNOWLEDGEMENTS}

We thank Alasdair J. Nisbet and Stewart Burgess (Moredun Research Institute) for stimulating discussions and support. This work was supported by the Research Foundation Flanders (FWO) [grant G009312N to TVL and grant G053815N to TVL and WD] and the Research Council (ERC) under the European Union's Horizon 2020 research and innovation program [grant 772026-POLYADAPT to TVL and 773902-SuperPests to TVL and JV]. WD is a post-doctoral fellow of the Research Foundation Flanders (FWO). Extended thanks to the following for contribution of European samples: Teresa Mateus, Veli Yilgör Çirak, Monique 
Mul, Cristian Magdaş, Susanne Kabell, Lise Roy, Miroslav Radeski, Tana Shtylla Kika, Antonio

Camarda, Danijela Horvatek Tomić, Martina Lichovnikova and José Francisco Lima Barbero.

\section{REFERENCES}

1 Abbas RZ, Colwell DD, lqbal Z, and Khan A, Acaricidal drug resistance in poultry red mite (Dermanyssus gallinae) and approaches to its management, Worlds Poult Sci J 70:113-124 (2014).

2 Tomley FM and Sparagano O, Spotlight on avian pathology: red mite, a serious emergent problem in layer hens, Avian Pathol 47:533-535 (2018).

3 Sigognault Flochlay A, Thomas E, and Sparagano O, Poultry red mite (Dermanyssus gallinae) infestation: A broad impact parasitological disease that still remains a significant challenge for the egg-laying industry in Europe, Parasit Vectors 10:4-9 (2017).

4 Mul M, Fact sheet: the poultry red mite, Dermanyssus gallinae (De Geer, 1778) A small pest that packs a big punch, Wagening UR (2013).

5 Arsenopoulos K, Agelou A, and Papadopoulos H, Dermanyssus gallinae - A ghost ectoparasite for Greek laying hen industry: Results of a preliminary study, 4th Panhellenic Conf Vet Product Anim Food Hyg, Volos, Greece (2017).

6 Pugliese N, Circella E, Marino M, De Virgilio C, Cocciolo G, Lozito P, et al., Circulation dynamics of Salmonella enterica subsp. enterica ser. Gallinarum biovar Gallinarum in a poultry farm infested by Dermanyssus gallinae, Med Vet Entomol 33:162-170 (2019).

7 Sparagano OAE, George DR, Harrington DWJ, and Giangaspero A, Significance and Control of the Poultry Red Mite, Dermanyssus gallinae, Annu Rev Entomol 59:447-466 (2014).

8 Thomas E, Chiquet M, Sander B, Zschiesche E, and Flochlay AS, Field efficacy and safety of fluralaner solution for administration in drinking water for the treatment of poultry red mite (Dermanyssus gallinae) infestations in commercial flocks in Europe, Parasit Vectors 10:1-9 (2017).

9 Gassel M, Wolf C, Noack S, Williams H, and Ilg T, The novel isoxazoline ectoparasiticide fluralaner: Selective inhibition of arthropod $\gamma$-aminobutyric acid- and I-glutamate-gated chloride channels and insecticidal/acaricidal activity, Insect Biochem Mol Biol 45:111-124 (2014).

10 Genchi C, Traldi G, and Hubert H, Efficacia della Flumetrina (Bayticol, Bayer) nel controllo dell' acaro rosso, Dermanyssus gallinae, De Geer 1778 [dei polli]., Arch Vet Ital 35:125-128 (1984).

11 Marangi M, Cafiero MA, Capelli G, Camarda A, Sparagano OAE, and Giangaspero A, Evaluation of the poultry red mite, Dermanyssus gallinae (Acari: Dermanyssidae) susceptibility to some acaricides in field populations from Italy, Exp Appl Acarol 48:11-18 (2009).

12 Zeman $\mathrm{P}$ and Železný J, The susceptibility of the poultry red mite, Dermanyssus gallinae (De Geer, 1778), to some acaricides under laboratory conditions, Exp Appl Acarol 1:17-22 (1985).

13 Beugnet F, Chauve C, Gauthey M, and Beert L, Resistance of the red poultry mite to pyrethroids in France, Vet Rec 140:577-579 (1997).

14 Nordenfors H, Höglund J, Tauson R, and Chirico J, Effect of permethrin impregnated plastic strips on Dermanyssus gallinae in loose-housing systems for laying hens, Vet Parasitol 102:121-131 (2001).

15 Zdybel J, Karamon J, and Cencek T, In vitro effectiveness of selected acaricides against red poultry mites (Dermanyssus gallinae, De Geer, 1778) isolated from laying hen battery cage farms localised in different regions of Poland, Bull Vet Inst Pulawy 55:411-416 (2011).

16 Marangi M, Morelli V, Pati S, Camarda A, Cafiero MA, and Giangaspero A, Acaricide residues in laying hens naturally infested by red mite Dermanyssus gallinae, PLoS ONE 7:3-8 (2012). 
17 Van Leeuwen T and Tirry L, Esterase-mediated bifenthrin resistance in a multiresistant strain of the two-spotted spider mite, Tetranychus urticae, Pest Manag Sci 63:150-156 (2007). Ay R and Gürkan MO, Resistance to bifenthrin and resistance mechanisms of different strains of the two-spotted spider mite (Tetranychus urticae) from Turkey, Phytoparasitica 33:237-244 (2005).

19 Dong K, Du Y, Rinkevich F, Nomura Y, Xu P, Wang L, et al., Molecular biology of insect sodium channels and pyrethroid resistance, Insect Biochem Mol Biol 50:1-17 (2014).

20 Van Leeuwen T and Dermauw W, The Molecular Evolution of Xenobiotic Metabolism and Resistance in Chelicerate Mites, Annu Rev Entomol 61:475-498 (2016).

21 Tan J, Liu Z, Wang R, Huang ZY, Chen AC, Gurevitz M, et al., Identification of Amino Acid Residues in the Insect Sodium Channel Critical for Pyrethroid Binding, Mol Pharmacol 67:513-522 (2005).

22 Tsagkarakou A, Van Leeuwen T, Khajehali J, Ilias A, Grispou M, Williamson MS, et al., Identification of pyrethroid resistance associated mutations in the para sodium channel of the two-spotted spider mite Tetranychus urticae (Acari: Tetranychidae), Insect Mol Biol 18:583-593 (2009).

23 Riga M, Bajda S, Themistokleous C, Papadaki S, Palzewicz M, Dermauw W, et al., The relative contribution of target-site mutations in complex acaricide resistant phenotypes as assessed by marker assisted backcrossing in Tetranychus urticae, Sci Rep 7:9202 (2017).

24 He H, Chen AC, Davey RB, Ivie GW, and George JE, Identification of a Point Mutation in the para-Type Sodium Channel Gene from a Pyrethroid-Resistant Cattle Tick, Biochem Biophys Res Commun 261:558-561 (1999).

25 Nyoni BN, Gorman K, Mzilahowa T, Williamson MS, Navajas M, Field LM, et al., Pyrethroid resistance in the tomato red spider mite, Tetranychus evansi, is associated with mutation of the para-type sodium channel, Pest Manag Sci 67:891-897 (2011).

26 Wu M, Adesanya AW, Morales MA, Walsh DB, Lavine LC, Lavine MD, et al., Multiple acaricide resistance and underlying mechanisms in Tetranychus urticae on hops, J Pest Sci 92:543-555 (2018).

27 Moyes CL, Vontas J, Martins AJ, Ng LC, Koou SY, Dusfour I, et al., Contemporary status of insecticide resistance in the major Aedes vectors of arboviruses infecting humans, PLoS Negl Trop Dis 11:e0005625 (2017).

28 Burgess STG, Bartley K, Nunn F, Wright HW, Hughes M, Gemmell M, et al., Draft Genome Assembly of the Poultry Red Mite, Dermanyssus gallinae, Microbiol Resour Announc 7:e01221-18 (2018).

29 Schicht S, Qi W, Poveda L, and Strube C, Whole transcriptome analysis of the poultry red mite Dermanyssus gallinae (De Geer, 1778), Parasitology 141:336-346 (2014).

30 Hall TA, BIOEDIT: a user-friendly biological sequence alignment editor and analysis program for Windows 95/98/ NT., Nucleic Acids Symp Ser 41:95-98 (1999).

31 Du Y, Garden DP, Wang L, Zhorov BS, and Dong K, Identification of new batrachotoxinsensing residues in segment IIIS6 of the sodium channel, J Biol Chem 286:13151-13160 (2011).

32 Rinkevich FD, Du Y, and Dong K, Diversity and convergence of sodium channel mutations involved in resistance to pyrethroids, Pestic Biochem Physiol 106:93-100 (2013).

33 Panini M, Anaclerio M, Puggioni V, Stagnati L, Nauen R, and Mazzoni E, Presence and impact of allelic variations of two alternative s-kdr mutations, M918T and M918L, in the voltagegated sodium channel of the green peach aphid Myzus persicae, Pest Manag Sci 71:878-884 (2014).

34 Karatolos N, Gorman K, Williamson MS, and Denholm I, Mutations in the sodium channel associated with pyrethroid resistance in the greenhouse whitefly, Trialeurodes vaporariorum, Pest Manag Sci 68:834-838 (2012). 
Major KM, Weston DP, Lydy MJ, Wellborn GA, and Poynton HC, Unintentional exposure to terrestrial pesticides drives widespread and predictable evolution of resistance in freshwater crustaceans, Evol Appl 11:748-761 (2018). Sabrina Bothorel, Céline Robert, Laurent Ruck, Julien Carpezat A, Resistance to pyrethroid insecticides in cabbage stem flea beetle (Psylliodes chrysocephala) and rape winter stem weevil (Ceutorhynchus picitarsis) populations in France, IOBC-WPRS Work Group "Integrated Control Oilseed Crop, ed. by Samantha M. Cook, Malgorzata Jedryczka IJ, Truman, and William, 89-104, Zagreb.

37 Kapantaidaki DE, Sadikoglou E, Tsakireli D, Kampanis V, Stavrakaki M, Schorn C, et al., Insecticide resistance in Trialeurodes vaporariorum populations and novel diagnostics for $\mathrm{kdr}$ mutations, Pest Manag Sci 74:59-69 (2017).

38 Kawada H, Higa Y, Komagata O, Kasai S, Tomita T, Nguyen TY, et al., Widespread distribution of a newly found point mutation in voltage-gated sodium channel in pyrethroid-resistant Aedes aegypti populations in Vietnam, PLoS Negl Trop Dis 3:e527 (2009).

39 Kasai S, Ng LC, Lam-Phua SG, Tang CS, Itokawa K, Komagata O, et al., First detection of a putative knockdown resistance gene in major mosquito vector, Aedes albopictus, Jpn J Infect Dis 64:217-221 (2011).

40 Hu Z, Du Y, Nomura Y, and Dong K, A sodium channel mutation identified in Aedes aegypti selectively reduces cockroach sodium channel sensitivity to type I, but not type II pyrethroids, Insect Biochem Mol Biol 41:9-13 (2011).

41 González-Cabrera J, Bumann H, Rodríguez-Vargas S, Kennedy PJ, Krieger K, Altreuther G, et al., A single mutation is driving resistance to pyrethroids in European populations of the parasitic mite, Varroa destructor, J Pest Sci 91:1137-1144 (2018).

42 Stara J, Pekar S, Nesvorna M, Kamler M, Doskocil I, and Hubert J, Spatio-temporal dynamics of Varroa destructor resistance to tau-fluvalinate in Czechia, associated with L925V sodium channel point mutation, Pest Manag Sci 75:1287-1294 (2018).

43 Alissandrakis E, llias A, and Tsagkarakou A, Pyrethroid target site resistance in Greek populations of the honey bee parasite Varroa destructor (Acari: Varroidae), J Apic Res 56:625-630 (2017).

44 Balvín $\mathrm{O}$ and Booth W, Distribution and Frequency of Pyrethroid Resistance-Associated Mutations in Host Lineages of the Bed Bug (Hemiptera: Cimicidae) Across Europe, J Med Entomol 55:923-928 (2018).

45 Morgan JAT, Corley SW, Jackson LA, Lew-Tabor AE, Moolhuijzen PM, and Jonsson NN, Identification of a mutation in the para-sodium channel gene of the cattle tick Rhipicephalus (Boophilus) microplus associated with resistance to synthetic pyrethroid acaricides, Int J Parasitol 39:775-779 (2009).

46 Wrzesin B, Czerwoniec A, and Wieczorek P, A survey of pyrethroid-resistant populations of Meligethes aeneus $\mathrm{F}$. in Poland indicates the incidence of numerous substitutions in the pyrethroid target site of voltage-sensitive sodium channels in individual beetles, Insect $\mathrm{Mol}$ Biol 23:682-693 (2014).

47 Vais $\mathrm{H}$, Williamson MS, Devonshire AL, and Usherwood PNR, The molecular interactions of pyrethroid insecticides with insect and mammalian sodium channels, Pest Manag Sci 57:877-888 (2001).

48 Usherwood PNR, Davies TGE, Mellor IR, O'Reilly AO, Peng F, Vais H, et al., Mutations in DIIS5 and the DIIS4-S5 linker of Drosophila melanogaster sodium channel define binding domains for pyrethroids and DDT, FEBS Lett 581:5485-5492 (2007).

49 Haddi K, Valbon WR, Viteri Jumbo LO, de Oliveira LO, Guedes RNC, and Oliveira EE, Diversity and convergence of mechanisms involved in pyrethroid resistance in the stored grain weevils, Sitophilus spp., Sci Rep 8:1-15 (2018).

50 Sparagano OAE and Giangaspero A, Parasitism in egg production systems: The role of the red mite (Dermanyssus gallinae), Improving the Safety and Quality of Eggs and Egg Products: Egg 
Chemistry, Production and Consumption, Woodhead Publishing Limited, pp. 394-414 (2011).

51 Grigoraki L, Pipini D, Labbé P, Chaskopoulou A, Weill M, and Vontas J, Carboxylesterase gene amplifications associated with insecticide resistance in Aedes albopictus: Geographical distribution and evolutionary origin, PLoS Negl Trop Dis 11:e0005533 (2017). 


\section{Figure Legends}

Figure 1. Mutations identified in segments of the PRM VGSC gene.

A) Nucleotide triplets present in the sequenced VGSC gene segments of PRM. The triplets and amino acids identified in the GRC1 and GRC2 strains that were tested with bioassays are underlined. Triplets/amino acids found in the SUSC strain are indicated in normal font, while those found in the screened populations are indicated in bold font. B) Schematic diagram of domain II and III of the VGSC. Mutations found in PRM are indicated with orange stars, while those found in other species and indicated with black circles. Those mutations that were functionally characterized in Xenopus are framed in a grey box. ${ }^{19}$

Figure 2. Map showing the origin of 53 PRM populations analysed in this study, spread across 15 European countries.

Figure 3. Schematic illustration of the distribution of pyrethroid resistance mutations in PRM populations across Europe. The classification of mutations was based on visual inspection of sequencing chromatographs and comprised three categories: 'absent', 'present' and 'fixed' (when no alternative signal was detected at the investigated position). 
Table 1. Toxicity of pyrethroid acaricides towards three PRM populations (SUSC, GRC1 and GRC2).

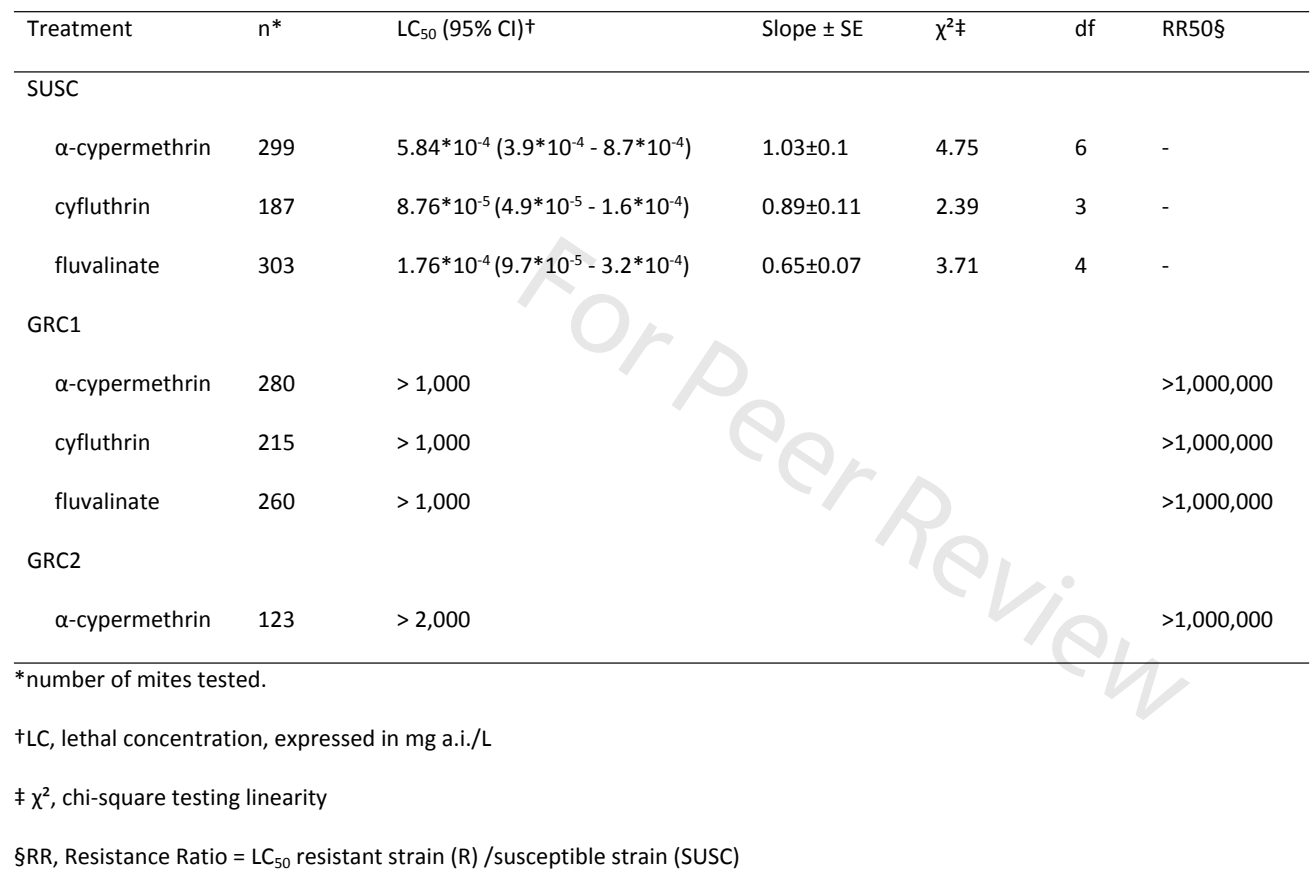


Table 2. Amino acid substitutions in two VGSC domains (II and III) of European PRM populations. The non- wild type alleles are indicated with bold font. A forward slash, separating amino acids, indicates that the allele is not fixed in a certain PRM population.

\begin{tabular}{|c|c|c|c|c|c|c|c|c|}
\hline \multirow[t]{2}{*}{ Country } & \multirow[t]{2}{*}{ Region } & \multirow[t]{2}{*}{ Code } & \multicolumn{4}{|c|}{ VGSC domain II } & \multicolumn{2}{|c|}{ VGSC domain III } \\
\hline & & & M918 & L925 & T929 & 1936 & F1534 & F1538 \\
\hline \multirow{2}{*}{ Germany } & Hannover & SUSC & $M$ & L & $\mathrm{T}$ & I & $F$ & $F$ \\
\hline & Hannover & GER1 & $\mathrm{M}$ & $\mathrm{L}$ & $\mathrm{T}$ & 1 & $\mathrm{~F}$ & $\mathrm{~F}$ \\
\hline \multirow{5}{*}{ Greece } & Megara & GRC1 & $\mathbf{L}$ & L & $T$ & 1 & $\mathbf{L}$ & $\mathrm{F}$ \\
\hline & Ilion & GRC2 & $\mathbf{L}$ & V & $\mathrm{T}$ & 1 & $\mathrm{~F}$ & $\mathrm{~F}$ \\
\hline & Megara & GRC3 & $\mathbf{L}$ & V & $\mathrm{T}$ & 1 & $F$ & $\mathrm{~F}$ \\
\hline & Megara & GRC5 & $\mathbf{L}$ & V & $\mathrm{T}$ & 1 & $\mathrm{~F}$ & $\mathrm{~F}$ \\
\hline & Avlona & GRC6 & $\mathbf{L}$ & v & $T$ & 1 & $\mathrm{~F}$ & $\mathrm{~F}$ \\
\hline \multirow{2}{*}{ Belgium } & Destelbergen & BEL1 & $M / L$ & $\mathrm{~L} / \mathrm{M}$ & $\mathrm{T}$ & I/F & $\mathrm{F} / \mathrm{L}$ & $\mathrm{F}$ \\
\hline & Evergem & BEL2 & $\mathbf{L}$ & $\mathrm{L}$ & $T$ & 1 & $\mathbf{L}$ & $\mathrm{F}$ \\
\hline \multirow{4}{*}{ Portugal } & Zezerovo & PRT1 & $\mathbf{L}$ & L & $\mathrm{T}$ & 1 & $\mathbf{L}$ & $\mathrm{F}$ \\
\hline & Souto da Carpalhosa & PRT2 & $\mathbf{L}$ & L & $\mathrm{T}$ & 1 & $\mathbf{L}$ & $\mathrm{F}$ \\
\hline & Riviera & PRT3 & $M / L$ & $\mathrm{~L}$ & $\mathrm{~T}$ & 1 & $F / L$ & $\mathrm{~F}$ \\
\hline & Casa de Hounance & PRT4 & $M / L$ & $\mathrm{~L}$ & $T$ & 1 & $\mathrm{~F} / \mathrm{L}$ & $\mathrm{F}$ \\
\hline \multirow{10}{*}{ UK } & Dungannon-Ireland & GBR1 & $\mathbf{L}$ & V & $T$ & 1 & $F$ & $\mathrm{~F}$ \\
\hline & Eastsussex & GBR2 & $\mathbf{L}$ & L & $T$ & 1 & $\mathbf{L}$ & $\mathrm{F}$ \\
\hline & Lincolnshire & GBR3 & $T / L$ & L & $T$ & 1 & $F / L$ & $\mathrm{~F} / \mathrm{L}$ \\
\hline & Durham & GBR4 & $\mathbf{L}$ & $\mathrm{L} / \mathrm{V}$ & $\mathrm{T}$ & 1 & $\mathrm{~F} / \mathrm{L}$ & $\mathrm{F}$ \\
\hline & Shropshire & GBR5 & $\mathrm{M} / \mathrm{T}$ & $\mathrm{L} / \mathrm{M}$ & $T$ & I & $\mathrm{F}$ & $\mathrm{F}$ \\
\hline & Dungannon-Ireland & GBR6 & $M / L$ & $\mathrm{~L}$ & $T$ & I/F & $\mathrm{F}$ & $\mathrm{F}$ \\
\hline & Kent & GBR7 & $\mathrm{M}$ & L & $\mathrm{T}$ & I/F & $\mathrm{F}$ & $\mathrm{F}$ \\
\hline & Suffolk & GBR8 & $\mathbf{L}$ & $\mathrm{L}$ & $\mathrm{T}$ & 1 & $F / L$ & $\mathrm{~F}$ \\
\hline & Cheshire & GBR9 & $T$ & $\mathrm{~L}$ & $\mathrm{~T}$ & 1 & $\mathrm{~F}$ & $\mathbf{L}$ \\
\hline & West Sussex & GBR10 & $\mathrm{M}$ & $\mathrm{L}$ & $\mathrm{T}$ & 1 & $\mathrm{~F}$ & $\mathrm{~F}$ \\
\hline \multirow{6}{*}{ Albania } & Peshkopi & ALB1 & $\mathbf{L}$ & L/V & $T$ & 1 & $F / L$ & $\mathrm{~F}$ \\
\hline & Lushnye & ALB2 & $\mathbf{L}$ & L/V & $T$ & I & $\mathrm{F} / \mathrm{L}$ & $\mathrm{F}$ \\
\hline & Shkoder & ALB3 & $\mathbf{L}$ & L/V & $\mathrm{T}$ & 1 & $\mathrm{~F} / \mathrm{L}$ & $\mathrm{F}$ \\
\hline & Durres & ALB4 & $\mathbf{L}$ & L/V & $\mathrm{T}$ & 1 & $F / L$ & $\mathrm{~F}$ \\
\hline & Berat & ALB5 & $\mathbf{L}$ & L/V & $\mathrm{T}$ & 1 & $\mathrm{~F} / \mathrm{L}$ & $\mathrm{F}$ \\
\hline & Korca & ALB6 & $\mathbf{L}$ & $\mathrm{L} / \mathrm{V}$ & $\mathrm{T}$ & 1 & $\mathrm{~F} / \mathrm{L}$ & $\mathrm{F}$ \\
\hline Czech & South Moravian region & CZE1 & $M / L$ & L/V & $\mathrm{T}$ & 1 & $\mathrm{~F}$ & $\mathrm{~F}$ \\
\hline Republic & Bohemia & CZE2 & M & $\mathrm{L} / \mathrm{M}$ & $T$ & $\mathbf{F}$ & $\mathrm{F}$ & $\mathrm{F}$ \\
\hline Turkey & Karacaali & TUR & $\mathrm{M}$ & $\mathrm{L}$ & 1 & 1 & $\mathrm{~F}$ & $\mathrm{~F}$ \\
\hline \multirow{3}{*}{ Spain } & Marchena, Seville & ESP1 & $\mathbf{L}$ & $\mathrm{L}$ & $T$ & 1 & $F / L$ & $\mathrm{~F}$ \\
\hline & Marchena, Seville & ESP2 & $\mathbf{L}$ & $\mathrm{L}$ & $\mathrm{T}$ & 1 & $F / L$ & $\mathrm{~F}$ \\
\hline & Marchena, Seville & ESP3 & $\mathbf{L}$ & $\mathrm{L}$ & $\mathrm{T}$ & 1 & $\mathrm{~F} / \mathrm{L}$ & $\mathrm{F}$ \\
\hline \multirow{4}{*}{ Croatia } & Zagreb & HRV1 & $M / L$ & $\mathrm{~L}$ & $\mathrm{~T}$ & 1 & F/L & $\mathrm{F}$ \\
\hline & Zagreb & HRV2 & $M$ & $\mathrm{~L}$ & $\mathrm{~T}$ & 1 & $F / L$ & $\mathrm{~F}$ \\
\hline & Zagreb & HRV3 & $M / L$ & L & $\mathrm{T}$ & 1 & $F / L$ & $\mathrm{~F}$ \\
\hline & Zagreb & HRV4 & $\mathrm{M} / \mathrm{L}$ & L/V & $\mathrm{T}$ & 1 & $\mathrm{~F} / \mathrm{L}$ & $\mathrm{F}$ \\
\hline \multirow{4}{*}{ Netherlands } & Lutten & NLD1 & $\mathrm{M} / \mathrm{L}$ & L/V & $\mathrm{T}$ & 1 & $F / L$ & $\mathrm{~F}$ \\
\hline & Barneveld & NLD2 & $\mathrm{M} / \mathrm{V}$ & $\mathrm{L}$ & $\mathrm{T}$ & 1 & $\mathrm{~F} / \mathrm{L}$ & $\mathrm{F}$ \\
\hline & Barneveld & NLD3 & $M / L$ & $\mathrm{~L}$ & $\mathrm{~T}$ & I & $F / L$ & $\mathrm{~F}$ \\
\hline & Aalten & NLD4 & $\mathrm{M}$ & $\mathrm{L}$ & $\mathrm{T}$ & 1 & $\mathrm{~F} / \mathrm{L}$ & $\mathrm{F}$ \\
\hline France & Grenade & FRA & $\mathrm{M} / \mathrm{T}$ & $\mathrm{L}$ & $T$ & 1 & $\mathrm{~F}$ & $\mathrm{~F} / \mathrm{L}$ \\
\hline \multirow{2}{*}{ Denmark } & Vejle & DNK1 & $M / L$ & L/V & $\mathrm{T}$ & I & $\mathrm{F}$ & $\mathrm{F}$ \\
\hline & Jylland & DNK2 & $\mathrm{M} / \mathrm{L}$ & $\mathrm{L} / \mathrm{V}$ & $\mathrm{T}$ & 1 & $\mathrm{~F}$ & $\mathrm{~F}$ \\
\hline \multirow{5}{*}{ Romania } & Tatarlaua & ROU1 & $\mathbf{L}$ & $\mathrm{L}$ & $T$ & 1 & $L$ & $\mathrm{~F}$ \\
\hline & Cuzdrioara & ROU3 & $M$ & $\mathrm{~L}$ & $\mathrm{~T} / \mathrm{I}$ & 1 & $\mathrm{~F}$ & $\mathrm{~F}$ \\
\hline & Cuzdrioara & ROU4 & $M$ & $\mathrm{~L}$ & $\mathrm{~T} / \mathrm{I}$ & 1 & $\mathrm{~F} / \mathrm{L}$ & $\mathrm{F}$ \\
\hline & Cuzdrioara & ROU5 & $M$ & $\mathrm{~L}$ & $T / I$ & 1 & $\mathrm{~F} / \mathrm{L}$ & $\mathrm{F}$ \\
\hline & Floresti & ROU6 & $\mathrm{M} / \mathrm{L}$ & $\mathrm{L}$ & $\mathrm{T}$ & $\mathrm{I}$ & $\mathrm{L}$ & $\mathrm{F}$ \\
\hline \multirow{2}{*}{ Italy } & Lecce & ITA1 & $M / L$ & L/V & $T$ & 1 & $\mathrm{~F}$ & $\mathrm{~F}$ \\
\hline & Cremona & ITA2 & $\mathbf{L}$ & v & $T$ & I & $\mathrm{F}$ & $\mathrm{F}$ \\
\hline
\end{tabular}


